\title{
DETERMINANTES DAS ESTRATÉGIAS INTERNACIONAIS DE ADAPTAÇÃO E DIFERENCIAÇÃO DE PME'S
}

\section{RESUMO}

É consenso na literatura que as circunstâncias do mercado induzem as firmas a promover estratégias de ajustes (adaptação e diferenciação) para atender às suas exigências. Todavia, o desafio para a academia consiste em determinar em que grau as firmas se adaptam ou diferenciam para exportar seus produtos. Desta forma, o presente estudo propõe-se evidenciar, por meio de um estudo empírico, como as características da firma e do mercado estrangeiro influenciam nas estratégias de exportação (adaptação e diferenciação) de empresas de pequeno e médio porte. Foram coletados dados de natureza primária e secundária, obtidos através que questionário semiestruturado, aplicado às PME’s mineiras e estes foram tratados por meio da Regressão Múltipla. Os resultados mostram que as estratégias de adaptação são influenciadas mais pelos condicionantes locais do país hospedeiro, ao passo que para as estratégias de diferenciação as características das firmas são mais relevantes.

Palavras-chave: Estratégias de Diferenciação. Estratégias de Adaptação. PME’s.

\section{DETERMINANTS OF ADAPTATION AND DIFFERENTIATION INTERNATIONAL STRATEGIES OF}

\section{SMES}

\begin{abstract}
There is a consensus that the market circumstances induce firms to promote strategies adjustments (adaptation and differentiation) to meet its requirements. However, the challenge for academia is to determine the degree to which firms adapt or differentiate their products to export. Thus, this study aims to demonstrate through an empirical study, which characteristics of the firm and the foreign market influence export strategies (adaptation and differentiation) of small and medium businesses. Data were obtained from primary and secondary nature, through semi-structured questionnaire administered to SMEs from Minas Gerais and these were treated by Multiple Regression. The results show that adaptation strategies are influenced more by local conditions of the host country whereas differentiation strategies are influenced by the firm specific characteristics.
\end{abstract}

Keywords: Differentiation Strategies. Adaptation Strategies. SME’s 


\section{FACTORES DETERMINANTES DE LAS ESTRATEGIAS INTERNACIONALES “ADAPTACIÓN Y DIFERENCIACIÓN" DE LAS PYME}

\section{RESUMEN}

Hay un consenso de que las circunstancias del mercado inducen a las empresas a promover estrategias de adaptación y ajustes (diferenciación) para cumplir sus requisitos. Sin embargo, el reto para el mundo académico es determinar el grado en que las empresas se adaptan o se diferencian para exportar sus productos. Así, este trabajo tiene como objetivo demostrar a través de un estudio empírico, cómo las características de la empresa y el mercado extranjero influencian sobre las estrategias de exportación (adaptación y diferenciación) de las pequeñas y medianas empresas. Fueron recogidos datos de carácter primario y secundario, que obtiene a través de cuestionario semiestructurado administrado a la Mineira PYME y éstas fueron tratados por medio de regresión múltiple. Los resultados muestran que las estrategias de adaptación está más influido por las condiciones locales del país anfitrión, mientras que las estrategias de diferenciación a las características de las empresas son más relevantes.

Palavras-clave: Estrategias de Adaptación. Estrategias de diferenciación. PYME.

Caroline Mendonça Nogueira Paiva ${ }^{1}$ Juciara Nunes de Alcântara ${ }^{2}$ Evandro Marcus Cintra ${ }^{3}$ Cristina Lelis Leal Calegario ${ }^{4}$

\footnotetext{
${ }^{1}$ Mestre em Administração pela Universidade Federal de Lavras - UFLA. Tutora à distância da Universidade Federal de São João Del-Rei - UFSJ. Brasil. E-mail: carolmn_be@yahoo.com.br

2 Doutoranda em Admnistração pela Universidade Federal de Lavras - UFLA. Brasil. E-mail: juciaranalcantara@gmail.com

3 Graduando em Admnistração pela Universidade Federal de Lavras - UFLA. Brasil. E-mail: evandro.cintra@live.com

${ }_{4}$ Doutora em Agricultural And Applied Economics pela University of Georgia, Estados Unidos. Professora da Universidade Federal de Lavras - UFLA. Brasil. E-mail: ccalegario@prpg.ufla.br
} 


\section{INTRODUÇÃO}

Grande parte da literatura internacional em marketing internacional tem focado a discussão entre adaptação e padronização como estratégias de exportação, conferindo especial ênfase ao desafio para encontrar o ponto ótimo entre as duas estratégias de marketing entre os diferentes países a fim de obter sucesso (Schmid \& Kotulla, 2011).

Embora muitos estudos não façam distinção entre as estratégias de adaptação e diferenciação (Schmid \& Kotulla, 2011), neste estudo as definições das estratégias seguem os conceitos apresentados em Axinn, Noordewier, \& Sinkula (1996) seguindo a definição de empresa multinacional e global. Para os autores, uma empresa multinacional ajusta seus produtos para cada país estrangeiro em um esforço para satisfazer as preferências dos consumidores locais. A empresa global, por sua vez, oferece o mesmo produto em qualquer país, oferecendo produtos padronizados de forma a alcançar um patamar mundial. A despeito das tendências de globalização, Theodosiou \& Leonidou (2003) afirmam que variações entre países em dimensões tais como as necessidades dos consumidores, condições de uso, poder de compra, infraestrutura comercial, cultura e tradições, leis e regulações, desenvolvimento tecnológico e saturação de mercado, exigem certo nível de ajustamento da estratégia da firma conforme as especificidades de cada mercado estrangeiro.

Desta forma, tem-se que as circunstâncias específicas do mercado induzem as firmas a promoverem pequenos ajustes em seu produto ou diferencia-lo de outros produtos para atender ao mercado e obtenção de grande volume de vendas pela exploração de diferentes necessidades dos consumidores através dos países (Theodosiou \& Leonidou, 2003). Assim, tendo em consideração as estratégias de ajustes (adaptação e diferenciação), o desafio para as pesquisas acadêmicas consiste em determinar sob quais condições e em que grau as firmas se adaptam ou se diferenciam (Theodosiou \& Leonidou, 2003).

Embora existam diversas teorias na literatura que explicam a atuação das firmas em mercados externos, como por exemplo, o paradigma eclético (Dunning, 1977), modelo de upsalla (Johanson e Vahlne, 1977), entre outros, a seleção de posições estratégicas das firmas para entrar em países estrangeiros, de uma forma geral, pode ser explicada com aporte teórico na Organização Industrial (OI), teoria institucional (TI) e na visão baseada em recursos (RBV) as quais definem que a estrutura industrial presentes no mercado bem como o conjunto de recursos da firma dirigem a posição estratégica. Assim, as duas primeiras se concentram na explicação da influência dos fatores externos à firma, ao passo que a terceira foca nos fatores internos e intrínsecos da firma.
De acordo com os pressupostos teóricos da Organização Industrial (OI) o mercado e a estrutura da indústria influenciam na definição da posição estratégica da firma, ou seja, sua conduta, os quais apresentam implicações positivas sobre seu desempenho (Zou \& Cavusgil, 2002). Assim, a posição estratégica da firma é definida em resposta às pressões do ambiente econômico em que ela está inserida, o que garante sua sobrevivência e desempenho (Porter, 1996). Desta forma, de acordo com os pressupostos da OI, o principal determinante do desempenho da firma são suas estratégias, que são definidas a partir das pressões das forças externas do ambiente e da indústria.

Derivada a partir dos fundamentos da OI, a Teoria Institucional (IT) postula que as instituições, definidas como as "regras do jogo", consistem nas restrições que estruturam as interações humanas (North, 1991; Peng, 2003) e são normalmente agrupadas em regras formais (leis e regulações) e restrições informais (costumes, normas e culturas). Desta forma, as diferenças nos vários níveis macro da sociedade, como as instituições regulatórias, os padrões sociais e as normas culturais entre os países, potencialmente condicionam as estratégias da firma (North, 1991).

A terceira perspectiva teórica, a visão baseada em recursos (RBV) sustenta que a heterogeneidade das firmas determinada pelo domínio de recursos raros, valiosos e de difícil substituição, mobilidade e imitação pelos concorrentes (Amit; Shoemaker, 1993), consiste em fontes de vantagem competitiva da firma (Barney, 1991; Prahalad \& Hamel, 1990; Teece, Pisano, \& Shuen, 1997). Desta forma, as estratégias da firma são moldadas a partir da combinação destes recursos específicos os quais geram melhor desempenho e vantagem frente aos concorrentes (Zou \& Cavusgil, 2002).

Neste contexto, o presente estudo propõe-se evidenciar, por meio de um estudo empírico, como as características da firma e do mercado estrangeiro influenciam a intensidade das estratégias de adaptação e diferenciação dos produtos de empresas de pequeno e médio porte. A partir das perspectivas teóricas supracitadas é possível entender e agrupar os fatores que influenciam e determinam as estratégias analisadas, as quais foram divididas em: (i) características específicas da firma (tamanho, experiência de exportação e comprometimento); e (ii) características do mercado de exportação (exigência do mercado, abertura e proximidade cultural), que serão explicitados nos tópicos a seguir. 


\section{CARACTERÍSTICAS ESPECÍFICAS DA FIRMA}

As características da firma apresentam grande influência em suas estratégias de exportação, pois correspondem ao conjunto de recursos que a firma possui que são a base para o desenvolvimento de uma estratégia organizacional e obtenção de vantagem competitiva sustentável no mercado (Ghedine, Zen, \& Prévot, 2010). Para atingir aos objetivos deste trabalho, serão analisadas três características específicas da firma: tamanho, experiência de exportação e comprometimento com a atividade.

O tamanho da firma geralmente consiste na quantidade de funcionários e gestores ligados a atividade de exportação representando a disponibilidade fatores, de domínio da firma, usados para alocação de recursos para tal operação (Katsikeas, 1994). Com isso, há um consenso de que grandes empresas apresentam mais recursos humanos e financeiros, possuem maior capacidade de produção, conseguem atingir economias de escala elevadas e por consequência enfrentam menores riscos em operações de exportação (Navarro, Acedo, Robson, Ruzo, \& Losada, 2010).

De fato, empresas maiores conseguem obter forças no mercado que podem ser utilizadas para extrair condições favoráveis em termos de custo de matéria-prima e capital (Lee \& Habte-Giorgis, 2004). Vantagens associadas com economias de escala e especialização, melhor acesso a recursos financeiros no mercado de capitais e melhores capacidades para assumir riscos, estão entre as razões que explicam por que grandes empresas tem melhor desempenho em mercados internacionais (Alvarez, 2004). Com isso, pressupõe-se que os produtos comercializados por empresas maiores, apresentam padrões mundiais tornando necessário pequenos ajustes ou adaptações para atuação em mercados distintos. Todavia, se necessário maiores ajustes, empresas maiores teriam maior facilidade tendo em vista as vantagens acima mencionadas.

Por outro lado, empresas menores possuem maior capacidade de flexibilização de seus processos e rotinas, sendo mais fácil acrescentar um diferencial em seus produtos. Muitas empresas de menor porte tem conseguido entrar em mercados externos diferenciando seus produtos em termos de marca e qualidade, entre outros atributos. Por não conseguirem produzir em ampla escala, os produtos das empresas menores são tidos como artesanais e desta forma se tornam especiais e valorizados no mercado internacional. Além disso, ainda existem apelos comerciais para consumir estes produtos artesanais como forma de ação social em mercados desenvolvidos. Assim, presume-se que:

H1-a: Quanto maior o tamanho da firma, maior é a adoção de estratégias de adaptação para ingressar no mercado externo;
H1-b: Quanto maior o tamanho da firma, menor é a adoção de estratégias de diferenciação para ingressar no mercado externo;

A experiência de exportação da firma referese ao tempo de atuação na atividade refletindo o nível de conhecimento sobre mercados estrangeiros (Navarro et al., 2010). Empresas experientes são mais propensas a identificar a demanda do mercado e possuem uma melhor compreensão de como se diferenciar no mercado externo, o que pode contribuir para melhor estimar as estratégias de exportação da firma (Gregory, Karavdic, \& Zou, 2007).

Ainda sobre esse entendimento, a experiência adquirida propicia aos gestores o conhecimento necessário para controlar o nível de envolvimento internacional da empresa (Freeman \& Cavusgil, 2007) já que exportadores inexperientes são mais propensos a precisarem de assistência para fortalecer seus recursos e obterem êxito no processo de internacionalização (Francis \& Collins-Dodd, 2000).

Exportadores estabelecidos podem usar a sua experiência, diferenciando os atributos do produto, tais como embalagens, serviço e garantia (Hultman, Robson, \& Katsikeas, 2009). Por outro lado, empresas com pouca experiência são induzidas a adaptarem seus produtos para atender as regras e normas do mercado externo. Assim, presume-se que empresas com maior experiência de exportação comportem-se estrategicamente conforme as hipóteses a seguir:

H2-a: Quanto menor a experiência de exportação da firma, maior é a adoção de estratégias de adaptação para ingressar no mercado externo;

H2-b: Quanto maior a experiência de exportação da firma, maior é a adoção de estratégias de diferenciação para ingressar no mercado externo;

Outro fator que influencia a adoção de estratégia da firma é o seu comprometimento ou continuidade da atividade de exportação. Este fator interfere diretamente sobre a alocação de recursos da firma para a atividade, ou seja, a firma direciona maior quantidade de recursos para exportação quando é comprometida com a atividade. Além disso, o comprometimento da firma com a exportação favorece o desenvolvimento das estratégias de forma continuada, conquistando, a cada venda, maior número de clientes e se fortalecendo no mercado estrangeiro.

Quanto mais recursos forem direcionados à exportação, maior a capacidade da firma em se colocar estrategicamente no mercado, diferenciando ou adaptando seus produtos buscando um ajustamento maior às necessidades dos diferentes mercados externos (Lages \& Montgomery, 2004). Os autores sugerem ainda, que o compromisso das empresas com a atividade de exportação, faz com que mais recursos sejam alocados para a atividade (aumentando seus custos) e, consequentemente, a empresa se torna capaz 
Determinantes das Estratégias Internacionais de Adaptação e Diferenciação de PME’S

de melhorar seus processos de planejamento e implementação de estratégias de adaptação e diferenciação (Lages \& Montgomery, 2004).

Uma firma comprometida com a atividade de exportação tende a manter sua estratégia, sendo de adaptação ou diferenciação. Sendo assim, levantam-se as seguintes hipóteses:

H3-a: Quanto maior o comprometimento da firma com a atividade de exportação, maior é a adoção de estratégias de adaptação para ingressar no mercado externo;

H3-b: Quanto maior o comprometimento da firma com a atividade de exportação, maior é a adoção de estratégias de diferenciação para ingressar no mercado externo;

\section{CARACTERÍSTICAS DO MERCADO DE EXPORTAÇÃO}

A adequação das estratégias de exportação ao contexto em que esta será implantada tem sido abordada por diversos estudos no campo de negócios internacionais como fator crucial para o desempenho da firma no exterior (Hultman et al., 2009). A configuração das forças presentes no mercado de exportação que influenciam as estratégias de exportação das firmas foi extraída a partir dos pressupostos da OI e da TI e foram analisadas conforme o grau de exigência dos consumidores no mercado externo, a abertura comercial e a proximidade do mercado externo.

Considerando o grau de exigência dos consumidores estrangeiros, sabe-se que a adaptação do produto é mais provável quando as condições econômicas prevalecentes do mercado de exportação são diferentes das do mercado doméstico (Hultman et al., 2009). Além disso, empresas cujo produto é mais influenciável pelo gosto dos clientes, favorecem a estratégia de diferenciação (Lages, Abrantes, \& Lages, 2008).

De forma semelhante, em mercados exigentes, os consumidores buscam por produtos mais elaborados, com alto valor agregado, pagando por eles um preço mais elevado por peças que tenham um diferencial (Padilha, Gallon, \& Mattos, 2012). Desta forma, tem-se que:

H4-a: Quanto maior a exigência do mercado de exportação, mais intenso deve ser o grau de adaptação da firma;

H4-b: Quanto maior a exigência do mercado de exportação, mais intenso deve ser o grau de diferenciação da firma;
Em mercados de maior abertura comercial, os condicionantes regulatórios são intensos, já que há grande necessidade de proteção dos consumidores, das empresas e sociedade (Hultman et al., 2009).

$$
\text { Diferenças nas regulamentações }
$$
governamentais, tais como normas técnicas dos produtos, leis de conteúdo local e políticas fiscais obrigam as empresas a adaptarem seus produtos (Lages et al., 2008) para ingressar no mercado externo. As pressões para a legitimidade em um mercado de exportação motivam um contrato de customização, a fim de facilitar a adaptação e diferenciação da empresa, mitigar diferenças entre o operador e o parceiro local, e assegurar a conformidade com o mercado (Yang, Su, \& Fam, 2012).

Em contrapartida, em mercados abertos, a entrada de grande variedade de produtos acirra a concorrência interna, sendo necessária às empresas exportadoras atuantes naquele mercado, uma maior diferenciação de seus produtos. Além da necessidade de adaptação dos produtos segundo os padrões técnicos, de saúde, ou de vigilância sanitária (Cavusgil, Chan, \& Zhang, 2003), torna-se necessário um maior grau de diferenciação para garantir as vendas em ambiente altamente competitivo.

Tendo em vista a abertura comercial do mercado, tem-se que:

H5-a: Quanto maior a abertura do mercado de exportação, maior é a adoção de estratégias de adaptação da firma para ingressar no mercado externo;

H5-b: Quanto maior a abertura do mercado de exportação, maior é a adoção de estratégias de diferenciação da firma para ingressar no mercado externo;

As diferenças entre os sistemas de valores, costumes, religião, níveis de educação dos consumidores, opiniões, tradições, normas e hábitos dos indivíduos em uma sociedade e causa impacto nas estratégias da firma podem apresentar impactos significantes nas preferências e padrões de uso dos consumidores (Pipkin, 2009)

A distância cultural também envolve diferenças de sistema jurídico de um país, incentivos, práticas administrativas e estilos de trabalho que dificultam o acesso a determinados mercados hospedeiros (Hofstede, 1980 apud Sakarya, Eckman, \& Hyllegard, 2007), necessitando de estratégias de adaptação. É através da estratégia de adaptação, que as empresas se adequam às diferenças culturais em termos de língua, gosto e hábitos de consumo (Lages et al., 2008) no mercado externo. Por outro lado, a similaridade entre mercados, verificada por meio da proximidade do mercado de origem com o mercado de exportação, implica em similaridade cultural, política, legal e econômica. Se o mercado de exportação é 
similar ao mercado de origem, as firmas podem ter vantagens de comunicação com os consumidores e governo local, menor custo de pesquisa de marketing, maior facilidade de negociação e de adaptação a regulações locais (Calantone et al, 2006). Desta forma, presume-se que em mercados com maior proximidade cultural, maior é a adoção de estratégias de diferenciação, para cativar o gosto dos consumidores locais. Espera-se neste trabalho, que a proximidade entre os países doméstico e hospedeiro, influenciem as estratégias da firma, conforme se segue:

H6-a: Quanto maior a proximidade do mercado de exportação com o mercado doméstico, menor deve ser o grau de adaptação da firma;

H6-b: Quanto maior a proximidade do mercado de exportação com o mercado doméstico, maior deve ser o grau de diferenciação da firma;

\section{METODOLOGIA}

Esta pesquisa de natureza quantitativa e descritiva buscou identificar como as estratégias de adaptação e de diferenciação de PMEs exportadoras do estado de Minas Gerais são influenciadas pelas características da firma e do mercado de exportação.

Os dados utilizados são de natureza primária e secundária, os quais foram obtidos nas bases de dados UnctadStat (2014) e World Data Bank (2014). Os dados primários foram coletados por meio de questionário semiestruturado aplicado às micro, pequenas e médias empresas exportadoras do estado de Minas Gerais. O pré-teste foi realizado com três empresas para afinar o instrumento de coleta de dados e eliminar os viesses de interpretação, sendo duas empresas exportadoras de Belo Horizonte e uma de Perdões, sul de Minas.

A população da presente pesquisa foi composta por pequenas e médias empresas, exportadoras do estado de Minas Gerais, que tiveram vendas internacionais no ano de 2010. O estado foi escolhido por dois motivos principais: a conveniência para os autores; e por ser o segundo estado com maior participação sobre o valor total exportado pelo país.

O critério adotado para classificação do porte das empresas é baseado no número de funcionários no qual, as micro empresas possuem até 19 funcionários, as pequenas empresas possuem de 20 a 99 funcionários, as médias empresas possuem de 100 até 499 funcionários (SEBRAE, 2014).

A relação das empresas exportadoras foi obtida pelo Cadastro Industrial de 2011 da FIEMG, cedido pelo Centro Industrial e Empresarial de Minas Gerais (CIEMG). Segundo o cadastro, em 2011 haviam 602 micro, pequenas e médias empresas exportadoras no estado. Deste total, no ano de 2010 (período de análise considerado para esta pesquisa), 68 não haviam exportado e 98 empresas não puderam ser contatadas (causas: atividades encerradas, inexistência do telefone ou não atendimento das ligações). Desta forma, a população final do estudo foi composta por 436 empresas exportadoras.

A coleta de dados foi feita em duas etapas: primeiramente as empresas foram contatadas por telefone e 98 questionários foram enviados via e-mail. Após 20 dias de espera, o retorno foi de apenas $14 \%$. Diante da baixa taxa de retorno, partiu-se para a segunda etapa de aplicação do questionário, por meio de visita in loco nas cidades mais próximas que continham uma grande quantidade de empresas exportadoras. Foram feitas visitas às cidades de Varginha, Três Corações, Santa Rita do Sapucaí, Itajubá, Nova Serrana, Divinópolis, São João Del Rei e Belo Horizonte. No total, foram aplicados 45 questionários, o equivalente a $10 \%$ da população, taxa de retorno compatível com outros estudos em Ciências Sociais. Assim, a amostra foi obtida por meio da amostragem não-probabilística por conveniência.

Visando dar maior confiabilidade à amostra do presente estudo, utilizou-se o Teste $t$ de amostra para verificar se havia diferenças significativas entre as médias da amostra e população para o número de funcionários. Optou-se pelo número de funcionários da firma (Tamanho), uma vez que esta variável está disponibilizada no cadastro industrial para toda a população. $O$ teste não apresentou diferenças significativas (p valor igual a 0,261), indicando que as médias da amostra $(112,95)$ e da população $(90,3)$ são semelhantes.

A unidade de análise adotada neste estudo foram os destinos de exportação das 45 empresas da amostra, sendo identificados 55 países para o ano de 2010, gerando um total de 166 observações.

Os resultados foram analisados por meio de regressão múltipla, estimando-se duas equações de regressão, nas quais a intensidade do uso das estratégias de diferenciação e de adaptação foram utilizadas como variáveis dependentes. Para estimar as equações, foi utilizado o software estatístico SPSS.

As equações de regressão podem ser apresentadas por:

$$
Y_{i t}=\beta_{1}+\beta_{2} X_{1 i t}+\beta_{3} X_{2 i t}+\ldots+\varepsilon
$$

Em que:

Y é a variável dependente (grau de intensidade do uso das estratégias de diferenciação e de adaptação);

Xn é a variável independente (características da firma e características do mercado de exportação);

$\beta 1$ é o intercepto;

$\beta n$ é o efeito linear de Xn; $\varepsilon$ é o erro.

As variáveis independentes foram categorizadas em dois grupos. No primeiro, foram agrupadas as variáveis relacionadas às características 
Determinantes das Estratégias Internacionais de Adaptação e Diferenciação de PME’S

específicas da firma (tamanho, experiência de exportação, e continuidade). $\mathrm{O}$ segundo grupo concentra as variáveis relacionadas às características do mercado de exportação (exigência do consumidor, o grau de abertura do mercado e a proximidade do mercado de exportação do país de origem da firma). A Tabela 1 apresenta os destinos de exportação, categorizados segundo o continente. Já a Tabela 2 sistematiza as variáveis utilizadas no modelo, sua mensuração e a fonte da qual foram retiradas.
Para atender aos pressupostos para o uso da estatística multivariada foram utilizados os testes de variância não-nula (teste ANOVA), a inexistência de multicolinearidade perfeita (teste VIF - tabela 6), homocedasticidade (testes Breusch-Pagan e Koenker), erros normalmente distribuídos (Teste de normalidade), autocorrelação dos resíduos (Teste Durbin-Watson tabela 6), conforme apontado por Gujarati (2006). Não houveram dados perdidos.

Tabela 1 -Destinos de Exportação.

\begin{tabular}{|c|l|}
\hline CONTINENTE & \multicolumn{1}{c|}{ PAÍSES } \\
\hline Ásia e Oceania & $\begin{array}{l}\text { Arábia Saudita, Austrália, China, Coréia do Sul, Japão, Polinésia Francesa, } \\
\text { Nova Caledônia, Nova Zelândia, Rússia, Tailândia, Taiwan. }\end{array}$ \\
\hline Europa & $\begin{array}{c}\text { Alemanha, Áustria, Bélgica, Dinamarca, Espanha, Finlândia, França, Grécia, } \\
\text { Holanda, Islândia, Itália, Noruega, Polônia, Portugal, Reino Unido, Rússia, } \\
\text { República Tcheca, Suíça. }\end{array}$ \\
\hline África & $\begin{array}{c}\text { África do Sul, Angola, Argélia, Cabo Verde, Camarões, Egito, Gana, Libéria, } \\
\text { Moçambique, Namíbia, Zâmbia. }\end{array}$ \\
\hline América do Norte e Central & Canadá, Costa Rica, EUA, México, Porto Rico, Trinidad e Tobago. \\
\hline América do Sul & $\begin{array}{c}\text { Argentina, Bolívia, Chile, Colômbia, Equador, Paraguai, Peru, Uruguai, } \\
\text { Venezuela. }\end{array}$ \\
\hline
\end{tabular}

Tabela 2 - Variáveis, mensuração e fonte dos dados.

\begin{tabular}{|c|c|c|}
\hline VARIÁVEL & MENSURAÇÃO & FONTE \\
\hline \multicolumn{3}{|l|}{$\underline{\text { Dependentes }}$} \\
\hline - Grau de diferenciação & $\begin{array}{l}\text { 1- Não diferencia } \\
2 \text { - Diferencia pouco } \\
3 \text { - Diferencia } \\
4 \text { - Diferencia muito }\end{array}$ & Primários \\
\hline - Grau de adaptação & $\begin{array}{l}1 \text { - Não adapta } \\
2 \text { - Adapta pouco } \\
3 \text { - Adapta } \\
4 \text { - Adapta muito }\end{array}$ & Primários \\
\hline \multicolumn{3}{|l|}{ Características da Firma } \\
\hline - Tamanho & Número de funcionários & Primários \\
\hline - Experiência de exportação da firma & Tempo de exportação & Primários \\
\hline
\end{tabular}


Determinantes das Estratégias Internacionais de Adaptação e Diferenciação de PME’S

\begin{tabular}{|c|c|c|}
\hline - Continuidade & $\begin{array}{l}1 \text { - Exporta todos os anos } \\
0 \text { - Não exporta todos os anos }\end{array}$ & Primários \\
\hline \multicolumn{3}{|c|}{ Características do Mercado de Exportação } \\
\hline - Exigência do consumidor & PIB/Pessoa empregada & World Bank \\
\hline - Abertura & (Exportações + Importações)/PIB & Unctad \\
\hline - Proximidade Cultural & $\begin{array}{l}1 \text { - Ásia e Oceania } \\
2 \text { - Europa } \\
3 \text { - África } \\
4 \text { - América do Norte e Central } \\
5 \text { - América do Sul }\end{array}$ & Categórica \\
\hline
\end{tabular}

\section{RESULTADOS E DISCUSSÕES}

Dentre as estratégias consideradas, a análise de frequência mostra que com relação ao grau de adaptação, $76 \%$ das empresas adaptam muito o produto enquanto $12 \%$ adaptam pouco e $11 \%$ somente adaptam. Com relação ao grau de diferenciação, os dados evidenciam que aproximadamente $65 \%$ das empresas diferenciam muito, enquanto que aproximadamente $21 \%$ somente diferenciam e $14,3 \%$ diferenciam pouco (Tabela 3 ).

Tabela 3 - Análise de Frequência das Variáveis

\begin{tabular}{|c|c|c|c|c|}
\hline \multirow{4}{*}{ Adaptação } & FREQUÊNCIA & $\%$ & \% CUMULATIVO \\
\cline { 2 - 5 } & Adapta pouco & 19,0 & 12,3 & 12,3 \\
\cline { 2 - 5 } & Adapta & 17,0 & 11,0 & 23,4 \\
\cline { 2 - 5 } & Adapta Muito & 118,0 & 76,6 & 100,0 \\
\hline \multirow{4}{*}{ Diferenciação } & Total & 154,0 & 100,0 & \\
\cline { 2 - 5 } & Diferencia pouco & 22,0 & 14,3 & 14,3 \\
\cline { 2 - 5 } & Diferencia & 32,0 & 20,8 & 17,5 \\
\hline \multirow{4}{*}{ Continuidade } & Diferencia Muito & 100,0 & 64,9 & 100,0 \\
\cline { 2 - 5 } & Total & 154,0 & 100,0 & \\
\cline { 2 - 5 } & Descontínua & 28,0 & 18,2 & 18,2 \\
\cline { 2 - 5 } & Contínua & 126,0 & 81,8 & 100,0 \\
\hline \multirow{4}{*}{$\begin{array}{c}\text { Proximidade } \\
\text { Cultural }\end{array}$} & Total & 154,0 & 100,0 & \\
\cline { 2 - 5 } & Ásia / Oceania & 21,0 & 13,6 & 13,6 \\
\cline { 2 - 5 } & Europa & 51,0 & 33,1 & 46,8 \\
\cline { 2 - 5 } & América do Norte e Central & 29,0 & 18,8 & 74,7 \\
\cline { 2 - 5 } & América do Sul & 39,0 & 25,3 & 100,0 \\
\cline { 2 - 5 } & Total & 154,0 & 100,0 & \\
\hline & & & & \\
\hline
\end{tabular}

Analisando estes dados em tabulação cruzada com a proximidade (Tabela 4), verificou-se que com relação à adaptação, $100 \%$ das empresas que adaptam pouco os produtos venderam para países da América do Sul, das empresas que adaptam, aproximadamente $65 \%$ venderam para países da Europa e 35\% atuaram na América do Norte. Entretanto, entre as empresas que adaptam muito houve maior dispersão dos países de 
Determinantes das Estratégias Internacionais de Adaptação e Diferenciação de PME’S

atuação, sendo que a maioria (34\%) exportaram para a Europa, $19,5 \%$ para a América do Norte e Central, $17,8 \%$ para a Ásia/Oceania e $16,9 \%$ atuaram na América do sul.

Quanto ao grau de diferenciação, verificou-se que entre as empresas que diferenciam pouco, $59 \%$ venderam para a Europa, $36 \%$ para América do Sul e $4,5 \%$ para a América do Norte. Para as empresas que apenas diferenciam, a maior parcela $(43,8 \%)$ exportou para o continente africano, e entre aquelas que diferenciam muito, $37 \%$ exportaram para a Europa.

Os resultados indicam que a Europa e a América do Norte exigem maior adaptação e também maior diferenciação das empresas, certamente devido à exigência dos consumidores e da elevada regulação e padrões institucionais. África e América do Sul, por sua vez, não exigem das empresas grandes adaptações e produtos ou processos, todavia requer considerável diferenciação para atuação.

Com relação aos resultados descritivos dos dados, relacionados na Tabela 5, torna-se relevante comentar apenas as variáveis dependentes e as referentes às características do mercado. $O$ grau de adaptação e de diferenciação das empresas é relativamente homogêneo, com desvio respectivo de 0,7 e 0,739 , indicando concentração entre os valores de 2 a 4, com média respectiva de 3,58 e 3,46.

Tabela 4 - Tabulação Cruzada das variáveis

\begin{tabular}{|c|c|c|c|c|c|c|c|c|c|}
\hline & \multicolumn{4}{|c|}{ ADAPTAÇÃO } & \multicolumn{4}{|c|}{ DIFERENCIAÇÃO } \\
\hline & & $\begin{array}{c}\text { Adapta } \\
\text { pouco }\end{array}$ & Adapta & $\begin{array}{c}\text { Adapta } \\
\text { Muito }\end{array}$ & Total & $\begin{array}{l}\text { Diferencia } \\
\text { pouco }\end{array}$ & Diferencia & $\begin{array}{l}\text { Diferencia } \\
\text { Muito }\end{array}$ & Total \\
\hline \multirow{5}{*}{ Regionalismo } & $\begin{array}{c}\text { Ásia / } \\
\text { Oceania }\end{array}$ & $0,0 \%$ & $0,0 \%$ & $17,8 \%$ & $13,6 \%$ & $0,0 \%$ & $3,1 \%$ & $20,0 \%$ & $13,6 \%$ \\
\hline & Europa & $0,0 \%$ & $64,7 \%$ & $33,9 \%$ & $33,1 \%$ & $59,1 \%$ & $3,1 \%$ & $37,0 \%$ & $33,1 \%$ \\
\hline & África & $0,0 \%$ & $0,0 \%$ & $11,9 \%$ & $9,1 \%$ & $0,0 \%$ & $43,8 \%$ & $0,0 \%$ & $9,1 \%$ \\
\hline & $\begin{array}{c}\text { América do } \\
\text { Norte e } \\
\text { Central } \\
\end{array}$ & $0,0 \%$ & $35,3 \%$ & $19,5 \%$ & $18,8 \%$ & $4,5 \%$ & $15,6 \%$ & $23,0 \%$ & $18,8 \%$ \\
\hline & $\begin{array}{l}\text { América do } \\
\text { Sul }\end{array}$ & $100,0 \%$ & $0,0 \%$ & $16,9 \%$ & $25,3 \%$ & $36,4 \%$ & $34,4 \%$ & $20,0 \%$ & $25,3 \%$ \\
\hline \multicolumn{2}{|c|}{ Total } & $100 \%$ & $100 \%$ & $100 \%$ & $100 \%$ & $100 \%$ & $100 \%$ & $100 \%$ & $100 \%$ \\
\hline \multirow[t]{2}{*}{ Continuidade } & \begin{tabular}{|c|}
$\begin{array}{c}\text { Descontí- } \\
\text { nuo }\end{array}$ \\
\end{tabular} & $31,6 \%$ & $23,5 \%$ & $15,3 \%$ & $18,2 \%$ & $9,1 \%$ & $28,1 \%$ & $17,0 \%$ & $18,2 \%$ \\
\hline & Contínuo & $68,4 \%$ & $76,5 \%$ & $84,7 \%$ & $81,8 \%$ & $90,9 \%$ & $71,9 \%$ & $83,0 \%$ & $81,8 \%$ \\
\hline \multicolumn{2}{|c|}{ Total } & $100 \%$ & $100 \%$ & $100 \%$ & $100 \%$ & $100 \%$ & $100 \%$ & $100 \%$ & $100 \%$ \\
\hline
\end{tabular}

Tabela 5 - Estatísticas Descritivas das variáveis

\begin{tabular}{|c|c|c|c|c|c|c|c|c|}
\hline & N & MÉDIA & MEDIANA & MODA & $\begin{array}{c}\text { DESVIO } \\
\text { PADRÃO }\end{array}$ & VARIÂNCIA & MÍNIMO & MÁXIMO \\
\hline Adaptação & 154 & 3,58 & 4,00 & 4 &, 703 &, 494 & 2 & 4 \\
\hline Diferenciação & 154 & 3,46 & 4,00 & 4 &, 739 &, 546 & 2 & 4 \\
\hline LN Tamanho & 154 & 4,34689 & 4,49981 & 4,500 & 1,200227 & 1,441 &, 693 & 5,991 \\
\hline LN Experiência & 154 & 2,66815 & 2,70805 & 2,708 &, 756809 &, 573 &, 693 & 4,605 \\
\hline Continuidade & 154 &, 82 & 1,00 & 1 &, 387 &, 150 & 0 & 1 \\
\hline $\begin{array}{c}\text { LN_PIB por pessoa } \\
\text { Empregada }\end{array}$ & 154 & 10,26994 & 10,37667 & 11,117 &, 736962 &, 543 & 7,711 & 11,117 \\
\hline $\begin{array}{c}\text { LN Abertura } \\
\text { Proximidade } \\
\text { Cultural }\end{array}$ & 154 & 12,77079 & 13,13889 & 15,247 & 1,861552 & 3,465 & 7,475 & 15,247 \\
\hline
\end{tabular}

Entre as características do mercado, verificouse que o PIB/pessoa empregada (exigência dos consumidores) apresentou média de 10,26 mil dólares, valor também homogêneo (desvio 0,54), e a abertura com média de 12,77 com maior desvio 1,86, indicando heterogeneidade entre os valores. 
A análise dos dados se deu através da técnica estatística Regressão Múltipla, cujos resultados apresentam-se na Tabela 6. Nesta tabela, é possível verificar também que os pressupostos para uso da estatística multivariada foram atendidos, através dos testes VIF (inexistência de multicolinearidade perfeita), Durbin-Watson (autocorrelação dos resíduos) e ANOVA (variância não nula). Para detectar presença de homocedasticidade foram realizados os testes Breusch-Pagan, cujo resultado foi 31,720, e Koenker, resultando 68,046, ambos com valores significativos para $\mathrm{p}<0,001$. Estes pressupostos foram analisados, conforme apontado em Gujarati (2006).

Embora os coeficientes de determinação múltiplos (R2) tenham apresentado valores abaixo (33\% e 20\%) do indicado por Gujarati (2006) nas equações estimadas, indicando limitação no poder de explicação de Y (estratégias de adaptação e diferenciação), entende-se que seria difícil obter neste coeficiente um valor adequado (acima de 60\%) dado a complexidade e dimensões multifacetadas das estratégias aqui analisadas.

$\mathrm{Na}$ equação 1, em que a estratégia de adaptação é utilizada como dependente, verifica-se apenas a significância das variáveis relativas às características do mercado de exportação, ou seja, exigência do consumidor, abertura comercial e proximidade, sendo observado uma relação negativa entre a proximidade e a adaptação.

Estes resultados confirmam as hipóteses $\mathrm{H} 4 \mathrm{a}$, relacionada à exigência do mercado, H5a referente à abertura comercial, H6a referente à proximidade cultural. Os resultados mostram que mercados exigentes aumentam o grau de adaptação da firma em $11 \%$, corroborando Lages et al. (2008) que afirma que a adaptação do produto é mais provável em mercados com consumidores de alto grau de exigência; e com Padilha et al. (2012), que coloca que em mercados exigentes os consumidores buscam por produtos mais elaborados e dentro das especificações exigidas pelo mercado.

Tabela 6 - Resultados das Equações de Regressão estimadas

\begin{tabular}{|c|c|c|c|c|}
\hline VARIÁVEIS INDEPENDENTES & ADAPTAÇÃO (1) & VIF (1) & DIFERENCIAÇÃO (2) & VIF (2) \\
\hline Constante & $3,675 * * *$ & & $3,367 * * *$ & \\
\hline \multicolumn{5}{|l|}{ Características Específicas da Firma } \\
\hline - Tamanho & 0,086 & 1,129 & $-0,009$ & 1,129 \\
\hline - Experiência de exportação da firma & 0,091 & 1,259 & $0,229 * *$ & 1,259 \\
\hline - Continuidade & $-0,091$ & 1,197 & $-0,289 *$ & 1,197 \\
\hline \multicolumn{5}{|l|}{ Características do Mercado de exportação } \\
\hline - PIB por pessoa empregada & $0,116^{*}$ & 1,971 & 0,062 & 1,971 \\
\hline - Abertura & $0,182 * *$ & 1,799 & $0,232 * *$ & 1,799 \\
\hline - Proximidade Cultural & $-0,179 * * *$ & 1,177 & $-0,051$ & 1,177 \\
\hline$R 2$ & 0,333 & & 0,204 & \\
\hline $\mathrm{F}$ & 11,829 & & 6,066 & \\
\hline F sig & 0,000 & & 0,000 & \\
\hline Durbin-Watson & 2,105 & & 1,897 & \\
\hline \multicolumn{5}{|l|}{ Teste ANOVA } \\
\hline $\mathrm{F}$ & $11,829 * * *$ & & $6,066 * * *$ & \\
\hline
\end{tabular}

Nota. $\mathrm{N}=154 ; * * *$ valores significativos $\mathrm{p}<0,001 ; * *$ valores significativos $\mathrm{p}<0,05$; * valores significativos $\mathrm{p}<0,10 ; \mathrm{n} / \mathrm{s}=$ não significativo.

A abertura comercial aumenta o grau de adaptação em 18\%, conforme Cavusgil et al. (2003), havendo necessidade de adaptação dos produtos conforme os padrões técnicos de saúde e vigilância sanitária. A proximidade, conforme se esperava, apresentou relação inversa ao grau de adaptação, ou seja, os mercados de exportação mais próximos reduzem o grau de adaptação das firmas em 17\%, uma vez que não existem grandes diferenças entre $o$ mercado de origem e o exportador. Os resultados são compatíveis com os encontrados em Kustin (2004).
Com relação à equação 2 , em que a variável dependente é a estratégia de diferenciação, verificou-se significância das variáveis experiência de exportação, continuidade e abertura comercial. As duas primeiras variáveis apresentaram influência positiva, enquanto a variável de Proximidade Cultural apresentou relação negativa. Estes resultados confirmam as hipóteses $\mathrm{H} 2 \mathrm{~b}$ e $\mathrm{H} 5 \mathrm{~b}$ e não confirma a hipótese $\mathrm{H} 3 \mathrm{~b}$ relativa ao comprometimento ou continuidade das firmas com exportação. Constata-se assim, que dentre as firmas analisadas, aquelas com maior experiência de exportação tendem a diferenciar seus produtos em 
Determinantes das Estratégias Internacionais de Adaptação e Diferenciação de PME’S

$22 \%$. Este resultado corrobora com a pesquisa de Hultman et al. (2009), a qual relatou que empresas experientes aumentam o grau de diferenciação de seus produtos principalmente nos atributos embalagem, serviços e garantia.

Ainda com relação à equação 2, estratégia de diferenciação, foi possível se confirmar a hipótese H5b em que países com maior abertura comercial exigem maior diferenciação da empresa para conquistar seu espaço naquele mercado. O resultado mostrou que a abertura do mercado aumenta o grau de diferenciação da firma em $23 \%$.

Todavia, ao contrário do que se esperava, constatou-se que a continuidade da firma na atividade de exportação reduz seu grau de diferenciação em $28 \%$. Isso permite supor que quanto mais comprometida a firma for com a exportação, ou seja, se ela exportar todos os anos, o seu produto passa a ser notado e por aquele mercado, exigindo menos esforço para diferenciar sua oferta. Este resultado diverge do encontrado por Lages \& Montgomery (2004) em seu estudo no qual a firma comprometida destina grande quantidade de recursos para se diferenciar ou se adaptar ao mercado de exportação.

Os resultados apresentados mostram que, na amostra e períodos considerados, para a adoção de estratégias de adaptação, apresentaram significância todas as variáveis que foram testadas, referentes às características do mercado. Este fato evidencia que, para as estratégias de adaptação, os condicionantes do mercado de exportação, suas exigências e regulamentações foram mais importantes do que as próprias características da firma. Todavia, isto não se confirma quando o grau de diferenciação é analisado, uma vez que se verificou maior influência das características da firma, sendo relevante apenas a abertura comercial devido a alta concorrência nestes mercados.

A variável abertura comercial foi medida pela soma das exportações e importações, dividido pelo PIB do país em questão (Tabela 2). Para esta variável, também pode-se observar um resultado interessante, já que ela foi significativa nas duas equações estimadas, mostrando que a abertura comercial do país destino é de extrema relevância para as estratégias da empresa exportadora, sendo que ao comparar os dois parâmetros (equação 1 e 2), verifica-se que abertura apresentou maior impacto sobre a estratégia de diferenciação (23\%), indicando que em ambientes altamente competitivos, as firmas precisam realmente ajustar seus produtos, em parte para atender às exigências do mercado, mas em maior parte para se diferenciar dos demais concorrentes.

Este resultado confirma as evidências encontradas na literatura (Calantone, Kim, Schmidt, \& Cavusgil, 2006; Theodosiou \& Leonidou, 2003) quanto à influência da abertura comercial sobre a estratégia de exportação, todavia, contribui para a literatura ao evidenciar que em ambientes competitivos a estratégia de diferenciação apresenta um maior peso que a de adaptação.

As demais hipóteses estimadas (H1a, H1b, H2a, H3a, H4b, H6b) não foram possíveis de ser confirmadas uma vez que não apresentaram significância nas equações estimadas, o que se constitui sugestões de pesquisa para estudos vindouros.

\section{CONSIDERAÇÕES FINAIS}

Esta pesquisa teve como objetivo central entender de que forma os fatores internos e externos à firma influenciam o grau de adoção das estratégias de exportação (adaptação e diferenciação) de pequenas e médias empresas exportadoras.

Embora a literatura em estratégias de exportação seja numerosa, verificou-se carência entre pesquisas que contemplam estudos em pequenas e médias empresas e de estudos que se engajam em investigar as condições e em que as firmas se adaptam ou se diferenciam. Este trabalho procurou responder parte das questões descritas acima, sem a pretensão de esgotar o assunto tratado, o que seria impossível neste trabalho dado a amplitude e complexidade das questões investigadas.

O trabalho apresenta contribuições interessantes ao campo, uma vez que se evidenciou que as estratégias de adaptação são influenciadas mais pelos condicionantes locais, como exigência dos consumidores, abertura comercial, e a proximidade cultural, ao passo que para as estratégias de diferenciação as características das firmas são mais relevantes. Assim, percebe-se que a estratégia de adaptação da firma é resultado das pressões e restrições mercadológicas ou institucionais sofridas nos mercados estrangeiros, são condições necessárias para a inserção e sobrevivência da firma naquele mercado.

O contrário pode ser observado com a estratégia de diferenciação, em que o único condicionante local relevante foi a abertura comercial do país, sugerindo que em mercados com grande abertura comercial, há muita concorrência e a firma então precisa de se destacar das demais atuantes naquele mercado. Todavia, para a definição desta estratégia, os fatores internos, foram mais relevantes, o que permite inferir que a estratégia de diferenciação se relaciona mais à disponibilidade da firma em seus comprometer recursos para atuar em mercados estrangeiros.

O estudo, no entanto apresenta algumas limitações. A primeira delas está relacionada ao tamanho da amostra, ocasionado pela baixa taxa de retorno dos questionários e o seu caráter não probabilístico. Outra limitação consiste na complexidade do fenômeno estudado, impossibilitando a realização de um modelo que abranja todas as variáveis possíveis que interferem na definição das estratégias estudadas. Além destas, constata-se a 
dificuldade em comparar realidades econômicas distintas, como países desenvolvidos e em estratégias são diferenciadas e são contingenciais, variando conforme as limitações das firmas e o contexto econômico do país.

Além das sugestões já mencionadas na seção de resultados, ou seja, confirmação das hipóteses que não se mostraram significativas neste trabalho, propõese que futuros estudos investiguem a natureza das estratégias de exportação, incluindo a estratégia de liderança em custos. Pode-se estudar como ocorre a

\section{REFERÊNCIAS}

Alvarez, R. (2004). Sources of export success in smalland medium-sized enterprises: the impact of public programs. International Business Review, 13(3), 383-400. doi:10.1016/j.ibusrev.2004.01.002

Axinn, C. N., Noordewier, T., \& Sinkula, J. M. . (1996). Export Strategies and Export Performance: an empirical investigation of a products/markets typology. In S. T. Cavusgil (Ed.), Advances in International Marketing (8th ed., p. 205). Connecticut: Greenwich.

Amit, R.; Shoemaker, P. (1993). Strategic Assets and Organizational Rents. Strategic Management Journal, 4: 33-46.

Barney, J. (1991). Firm Resources and Sustained Competitive Advantage. Journal of Management, 17(1), 99-120. doi:10.1177/014920639101700108

Calantone, R. J., Kim, D., Schmidt, J. B., \& Cavusgil, S. T. (2006). The influence of internal and external firm factors on international product adaptation strategy and export performance: A three-country comparison. Journal of Business Research, 59(2), 176-185. doi:10.1016/j.jbusres.2005.05.001

Cavusgil, S. T., Chan, K., \& Zhang, C. (2003). Strategic Orientations in Export Pricing: A Clustering Approach to Create Firm Taxonomies. Journal of International Marketing, 11(1), 47-72. doi:10.1509/jimk.11.1.47.20136

Dunning, J. (1977). Trade, location of economic activity, and the multinational enterprise: a search for an eclectic approach. In: OHLIN, B. et al (ed.) The international allocation of economic activity. London: Macmillan.

Francis, J., \& Collins-Dodd, C. (2000). The Impact of Firms' Export Orientation on the Export Performance of High-Tech Small and MediumSized Enterprises. Journal of International Marketing, 8(3), 84-103. doi:10.1509/jimk.8.3.84.19631 desenvolvimento, de forma que acredita-se que as

definição destas estratégias, se de forma emergente ou deliberada, ou referente ao processo de implementação da estratégia, em que momento uma estratégia deliberada recebe ajustes emergentes. Futuros estudos também devem ampliar os fatores explicativos, considerando a contingência das firmas em diferentes mercados.

Freeman, S., \& Cavusgil, S. T. (2007). Toward a Typology of Commitment States Among Managers of Born-Global Firms: A Study of Accelerated Internalization. Journal of International Marketing, 15(4), 1-40.

Ghedine, T., Zen, A. C., \& Prévot, F. (2010). A Influência da Internacionalização no Desenvolvimento de Recursos e Capacidades Vinculadas à Estratégia de Operações da Firma: O Caso Marcopolo S.A. SIMPOI - Simpósio de Adminsitração Da Produção, Logística E Operações Internacionais, 13, 1-16.

Gregory, G., Karavdic, M., \& Zou, S. (2007). The Effects of E-Commerce Drivers on Export Marketing Strategy. Journal of International Marketing, 15(2), 30-57.

Gujarati, D. N. . (2006). Economietria Básica (p. 812). Rio de Janeiro: Elsevier.

Hultman, M., Robson, M. J., \& Katsikeas, C. S. (2009). Export Product Strategy Fit and Performance: An Empirical Investigation. Journal of International Marketing, 17(4), 1-23. doi:10.1509/jimk.17.4.1

Johanson, J.; Vahlne, J. (1977). The internationalization process of the firm: a model of knowledge and increasing foreign market commitment. Journal of International Business Studies, v. 8, n. 1, p. 23-32.

Katsikeas, C. S. (1994). Export Competitive Advantages: The Relevance of Firm Characteristics. International Marketing Review, 11(3), 33-53.

Kustin, R. A. (2004). Marketing mix standardization: a cross cultural study of four countries. International Business Review, 13(5), 637-649. doi:10.1016/j.ibusrev.2004.07.001

Lages, L. F., Abrantes, J. L., \& Lages, C. R. (2008). The STRATADAPT scale: A measure of marketing strategy adaptation to international business 
Determinantes das Estratégias Internacionais de Adaptação e Diferenciação de PME’S

markets. International Marketing Review, 25(5), 584-600. doi:10.1108/02651330810904107

Lages, L. F., \& Montgomery, D. B. (2004). Export performance as an antecedent of export commitment and marketing strategy adaptation: Evidence from small and medium-sized exporters. European Journal of Marketing, 38(9/10), 11861214. doi:10.1108/03090560410548933

Lee, J., \& Habte-Giorgis, B. (2004). Empirical approach to the sequential relationships between firm strategy, export activity, and performance in U.S. manufacturing firms. International Business Review, 13(1), 101-129. doi:10.1016/j.ibusrev.2003.05.003

Navarro, A., Acedo, F. J., Robson, M. J., Ruzo, E., \& Losada, F. (2010). Antecedents and Consequences of Firms' Export Commitment: An Empirical Study. Journal of International Marketing, 18(3), 41-61.

North, D. C. (1991). Institutions. Journal of Economic Perspective, 5(1), 97-112.

Padilha, A. C. M., Gallon, C., \& Mattos, P. de. (2012). Fatores condicionantes da implementação de estratégias competitivas e cooperativas: o caso das indústrias de joias de Guaporé-RS. Revista IberoAmericana de Estratégia - RIAE, 11(2), 34-69.

Peng, M. W. (2003). Institutional Transitions and Strategic Choices, (1997), 275-296.

Pipkin, A. (2009). Marketing Internacional: uma abordagem estratégica. (Aduaneiras, Ed.) (3rd ed.). São Paulo: Aduaneiras.

Porter, M. E. (1996). What is strategy? Harvard Business Review, 74(6), 61-78. doi:10.1098/rspb.2008.0355

Prahalad, C. K., \& Hamel, G. (1990). The Core Competence of the Corporation. Harvard Business Review, 68(3), 79-91. doi:10.1007/3-540-30763X_14

Sakarya, S., Eckman, M., \& Hyllegard, K. H. (2007). Market selection for international expansion: Assessing opportunities in emerging markets.
International Marketing Review, 24(2), 208-238. doi:10.1108/02651330710741820

Schmid, S., \& Kotulla, T. (2011). 50 years of research on international standardization and adaptationFrom a systematic literature analysis to a theoretical framework. International Business Review, 20(5), 491-507. doi:10.1016/j.ibusrev.2010.09.003

SEBRAE. (2014). Critérios e conceitos para classificação de empresas. Retrieved January 10, 2014, from http://www.sebrae.com.br/uf/goias/indicadores-dasmpe/classificacao-empresarial/criterios-e-conceitospara-classificacao-de-empresas/criterios-econceitos-para-classificacao-de-empresas

Teece, D. J., Pisano, G., \& Shuen, A. (1997). Dynamic capabilities and strategic management. Strategic Management Journal, 18(7), 509-533. doi:10.1002/(SICI)10970266(199708)18:7<509::AID-SMJ882>3.0.CO;2-Z

Theodosiou, M., \& Leonidou, L. C. (2003). Standardization versus adaptation of international marketing strategy: an integrative assessment of the empirical research. International Business Review (Vol. 12, pp. 141-171). doi:10.1016/S09695931(02)00094-X

UnctadStat. (2014). Indicadores. Retrieved October 14, 2013, from http://unctadstat.unctad.org/ReportFolders/reportFo lders.aspx?sCS_referer=\&sCS_ChosenLang=en

World Data Bank. (2014). World Development Indicators. Retrieved October 15, 2013, from http://databank.worldbank.org/data/views/variables election/selectvariables.aspx?source=worlddevelopment-indicators\#

Yang, Z., Su, C., \& Fam, K. (2012). Dealing with Institutional Distances in International Marketing Channels: Governance Strategies That Engender Legitimacy and Efficiency. Journal of Marketing, 76(May), 41-55.

Zou, S., \& Cavusgil, S. T. (2002). The GMS: A Broad Conceptualization of Global Marketing Strategy and Its Effect on Firm Performance. Journal of Marketing, 66(October), 40-56. 